\title{
Efficient and stable holographic gratings stored in an environmentally friendly photopolymer
}

Marta Morales-Vidal, Manuel G. Ramírez, Daniel Sirvent, Francisco J. Martínez-Guardiola, Mariela L. Álvarez, et al.

Marta Morales-Vidal, Manuel G. Ramírez, Daniel Sirvent, Francisco J. Martínez-Guardiola, Mariela L. Álvarez, Inmaculada Pascual, "Efficient and stable holographic gratings stored in an environmentally friendly photopolymer," Proc. SPIE 11207, Fourth International Conference on Applications of Optics and Photonics, 112072J (3 October 2019); doi: 10.1117/12.2530574

SPIE Event: IV International Conference on Applications of Optics and Photonics (AOP 2019), 2019, Lisbon, Portugal 


\title{
Efficient and stable holographic gratings stored in an environmentally friendly photopolymer
}

\author{
Marta Morales-Vidal ${ }^{* a}$, Manuel G. Ramírez ${ }^{\mathrm{b}}$, Daniel Sirvent ${ }^{\mathrm{b}}$, Francisco J. Martínez ${ }^{\mathrm{c}}$, \\ Mariela L. Álvarez ${ }^{c}$, Inmaculada Pascual ${ }^{\text {b, }}$ \\ a.U. Física Aplicada a las Ciencias y las Tecnologías, Universidad de Alicante, Ap. de correos 99, \\ Alicante E-03080, Spain; \\ bDepartamento de Óptica, Farmacología y Anatomía, Universidad de Alicante, Ap. de correos 99, \\ Alicante E-03080, Spain; \\ ${ }^{\mathrm{c}}$ Departamento de Física, Ingeniería de Sistemas y Teoría de la Señal, Universidad de \\ Alicante, Ap. de correos 99, Alicante E-03080, Spain;
}

\begin{abstract}
Holographic gratings stored in low-toxicity photopolymer, Biophotopol, have been analyzed to achieve stable and efficient holograms. A curing process allows the hologram stabilization, but at the same time, it could produce a diffraction efficiency $(D E)$ reduction. Here, a detailed low-cost LED curing protocol is shown to stabilize over time $1205 \mathrm{l} / \mathrm{mm}$ transmission holograms, and at the same time, a 33\% DE increment (with respect non-curing holograms) have been demonstrated. Finally, to obtain a better understanding of $D E$ change, a theoretical fit of our experimental result, based on Kogelnik's coupled wave theory was carried out and discussed.
\end{abstract}

Keywords: Holography, holographic recording materials, photopolymers, low-toxicity photopolymer, LED-curing

\section{INTRODUCTION}

Phase holograms have been recorded on several sensitive materials: silver emulsions, dichromated gelatin photoresists, photorefractive polymers or photopolymers. From all of them, photopolymers present important advantages like the ability to self-developing, dry-processing availability, good stability on thick emulsion layers, high sensitivity, high diffraction efficiency and high resolution. Nowadays, the development of green technologies has a huge impact on society. For this reason, our research group patented a water-soluble and acrylate-based photopolymer. This material, called Biophotopol, is low toxicity and has good recycling properties. It has been used to prepare transmission ${ }^{1,2,3}$ and reflection gratings ${ }^{4}$, as well as holographic lenses ${ }^{5}$. Hologram gratings with high maximum diffraction efficiency $D E_{\max }$ are preferred, whereas also it is very important that gratings remain over time. The dye of the photopolymer is consumed in the exposed areas, but it is not consumed at non-exposed areas. Then, the photopolymerization reaction could continue if the hologram is exposed to ambient light at initially non-exposed and non-polymerizated zones. Therefore, the hologram progressively diminishes its $D E_{\max }$ and its response would be not reproducible over time. A curing stage after recording the holograms, allow to remove the concentration gradient and provide temporal stability to the holographic gratings ${ }^{6,7}$. However, a curing stabilization stage could produce a $D E_{\max }$ decrement $^{6}$.

A curing stage to stabilize over time the holograms recorded in this material has been previously realized by our group ${ }^{4,5}$, also it has been demonstrated that LED curing stage can produce around $10 \% D E$ increment $^{4}$ in reflection holographic gratings. In this work, it is achieved around a 30\% DE increment in transmission holographic gratings after performing a low-cost curing stabilization stage upon non-overmodulated holograms ${ }^{8}$. The experimental results have been supported with a theoretical analysis based on Kogelnik's coupled wave theory9. This theory provides a useful approach to the parameters that determine the efficiency of the grating. $D E_{\max }$ at Bragg condition is determined by $v$ parameter which mainly depends by $\Delta n \times d$ product. Slant factors are $\mathrm{c}_{\mathrm{r}}$ and $\mathrm{c}_{\mathrm{s}}$, while $\lambda_{0}$ is the recording wavelength.

$$
v=i \frac{\pi \cdot \Delta n \cdot d}{\lambda \sqrt{c_{r} \cdot c_{s}}}
$$

*marta.morales@ua.es; phone 965903400

Fourth International Conference on Applications of Optics and Photonics, edited by

Manuel F. M. Costa, Proc. of SPIE Vol. 11207, 112072 J • (c) 2019 SPIE

CCC code: $0277-786 \mathrm{X} / 19 / \$ 21 \cdot$ doi: $10.1117 / 12.2530574$ 


\section{METHODOLOGY}

\subsection{Photopolymer material and curing stage}

Holographic gratings were stored over optimized Biophotopol (average refractive index, $n=1.5$ ). The solution composition in water was: $13.0 \mathrm{w} / \mathrm{w} \%$ poly(vinyl alcohol) (PVA) as inert binder polymer, $0.39 \mathrm{M}$ sodium acrylate $(\mathrm{NaAO})$ as polymerizable monomer, $9.0 \times 10^{-3} \mathrm{M}$ triethanolamine (TEA) as coinitiator and plasticizer and $1.0 \times 10^{-3} \mathrm{M}$ sodium salt $5^{\prime}$-riboflavin monophosphate $(\mathrm{RF})$ as sensitizer dye. The solution was deposited over levelled glass substrates $(6.3 \times 6.3$ $\mathrm{cm}$ ) and dried in an incubator (Climacell 111), with controlled relative humidity and temperature $\left(60 \pm 5 \%\right.$ and $20 \pm 1^{\circ}$ ), during $21 \mathrm{~h}$. After recording, a 20 min curing stage with a LED lamp emitting at the visible spectrum, from 400 to $750 \mathrm{~nm}$, $\left(13.5 \mathrm{~W}, 875 \mathrm{~lm}\right.$ at $6500 \mathrm{~K}, 30^{\circ}$, Lexman) was carried out to ensure stability over time in some holograms.

\subsection{Holographic setup}

Transmission holograms were recorded by the interference between two coherent beams (object and reference) at the photopolymer layer, reaching symmetrically by the same side. A continuous (CW) Argon Ion Laser 2060-10S S/N 944 (Spectra-Physics Beam Lok) emitting at $\lambda_{0}=488 \mathrm{~nm}$ (at which RF is sensitive) was split into two secondary beams with an intensity ratio $1: 1$. Both replicas were spatially filtered and collimated to yield a plane-wave of $1.5 \mathrm{~cm}$ beam diameter $(\phi)$. Two replicas of $3.3 \mathrm{~mW}$ imping to the photopolymer at a recording angle $\theta_{\mathrm{o}}=\theta_{\mathrm{r}}=17.1^{\circ}$ with respect to normal incidence (Fig. 1a), which corresponds with $1205 \mathrm{l} / \mathrm{mm}$ grating frequency. The exposure times were adjusted to obtain different exposure values. Holographic transmitted gratings illuminated over white light is shown in Fig. 1b. One advantage of these gratings versus the ones recording in symmetric holographic reflection setup, is the possibility to evaluate, in real time, the transmitted and diffracted intensity beam relation by using a He-Ne laser at $632.8 \mathrm{~nm}$ (at which $\mathrm{RF}$ is not sensitive) while recording the grating with an Argon laser $(488 \mathrm{~nm}$ ), see Fig. 1c. After recording (and after curing stage, if proceed) the hologram reconstruction was performed by spinning the sample with a monitored platform around its vertical axis around Bragg's angle. The diffraction and transmitted intensities of a He-Ne laser beam that impinges at the recorded gratings, were registered at different reconstruction angles.

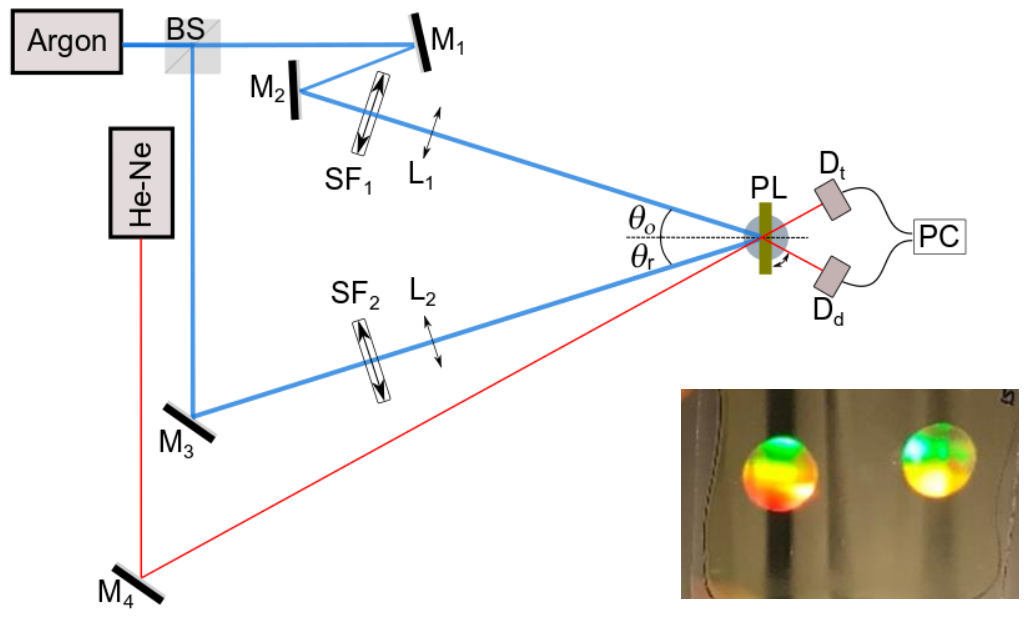

a)

b)

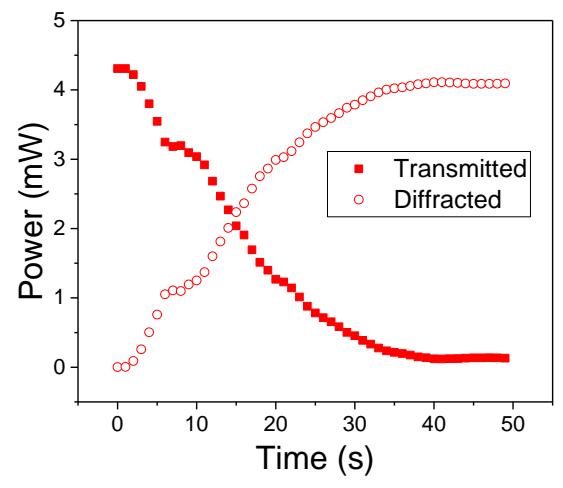

c)

Figure 1. a) Recording and reconstructing transmission holographic setup. BS: beam splitter; SF objective and pinhole); $\mathrm{M}_{\mathrm{i}}$ : mirrors; $\mathrm{L}_{\mathrm{i}}$ : lenses; $\theta_{\mathrm{o}}$ and $\theta_{\mathrm{r}}$ : object and reference recording angles; PL: photopolymer layer; $\mathrm{D}_{\mathrm{t}}$ : power detector of transmitted red beam; Dd: power detector of diffracted red beam; PC: Computer. b) Holographic transmitted gratings illuminated over a white light. c) Transmitted and diffracted intensity beams of a He-Ne laser.

\subsection{Data analysis}

Experimental $D E$ values were obtained as the ratio between diffracted and incident beam power, measured at different impinge angles to the photopolymer. The Kogelnik's coupled-wave theory gives analytical solutions of the holographic 
$D E$ properties9. In this work, the $D E$ values as an angular function were fitted by means of Kogelnik's theory and thus refractive index $(\Delta n)$, effective optical thickness $(d)$ and refractive index $(n)$ parameters were obtained.

\section{RESULTS}

\subsection{Diffraction efficiency before and after curing treatment}

Here, it has been studied the $D E$ of transmission gratings recording at different time exposures (before and after performing a curing treatment). From the analysis of the experimental results, we demonstrate that in a grating recorded at $0.07 \mathrm{~J} / \mathrm{cm}^{2}$ a curing treatment produces a 33\% DE increment. However, in a grating recorded at higher exposures $\left(H=0.16 \mathrm{~J} / \mathrm{cm}^{2}\right) \mathrm{we}$ observe a $D E$ decrement after a curing treatment (Fig. 2). In next section, a theoretical fit will be performed to explain why a curing stage increases $D E_{\max }$ in gratings recorded at low exposures $\left(0.02\right.$ and $\left.0.07 \mathrm{~J} / \mathrm{cm}^{2}\right)$ while in gratings recorded at higher exposures $\left(0.16 \mathrm{~J} / \mathrm{cm}^{2}\right)$ is shown the opposite effect.
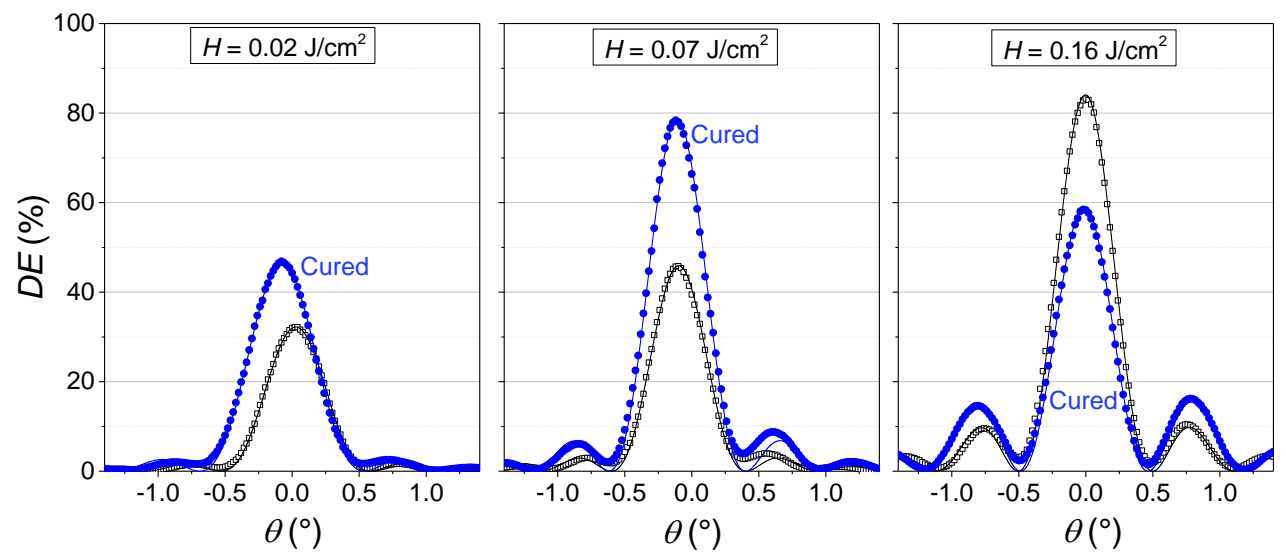

Figure 2. Diffraction efficiency $(D E)$ from holograms recording at different exposures as a function of the reconstruction angle before and after curing treatment.

\subsection{Kogelnik's theory and overmodulation effect}

The aim of this section is to give theoretical support to our experimental work, explaining why the curing stage must be performed after a particular exposure time. From Kogelnik's theory, we can fit experimental values (squares and circles) shown in Figure 2, obtaining a theoretical fit before and after the curing stage (black and blue line, respectively). This analysis allows us to obtain a better understanding of holographic parameters at different conditions in cured or non-cured holograms. Experimental $D E_{\max }$, as well as some parameters obtained from Kogelnik's fit, are shown in Table 1.

Accuracy fit is demonstrated by the comparison between experimental and fitted $D E_{\max }$ values, as well as seeing very similar $n$ values obtained from different fits before curing. Parameter trends before and after curing at different recording intensities is shown in Table 1. From these trends, we would like to remark $\Delta n \times d$ product in high exposure conditions. A highest $\Delta n=3.4 \times 10^{-3}$ is observed after curing the high exposure intensity hologram $\left(0.16 \mathrm{~J} / \mathrm{cm}^{2}\right)$. However, it is not observed the highest $D E$ (at this high exposure conditions) as we could expect ${ }^{4}$ because the highest $\Delta n \times d$, so probably after curing this grating, overmodulation effects appear. In order to confirm this hypothesis, we can observe Figure 1c. In the figure, was shown the He-Ne diffracted intensity vs time along $50 \mathrm{~s}$ recording time. As we can observe in that figure, the maximum diffraction intensity is achieved after around $40 \mathrm{~s}$, while higher exposure time does not mean higher diffraction intensities. From the analyzed results is concluded that overmodulation effects appear when gratings are recorded at higher exposures, but avoiding this situation, a curing stage allows to obtain stable over time and more efficient gratings. 
Table 1. Experimental $D E_{\max }$ parameter and fitted parameters obtained from the reconstructed hologram data.

\begin{tabular}{|c|c|c|c|c|c|c|c|}
\hline \multirow{2}{*}{\multicolumn{2}{|c|}{$\begin{array}{c}\text { Experimental/Fitted } \\
\text { Parameter }\end{array}$}} & \multicolumn{2}{|c|}{$H=0.02 \mathrm{~J} / \mathrm{cm}^{2}$} & \multicolumn{2}{|c|}{$H=0.07 \mathrm{~J} / \mathrm{cm}^{2}$} & \multicolumn{2}{|c|}{$H=0.16 \mathrm{~J} / \mathrm{cm}^{2}$} \\
\hline & & \multirow{2}{*}{\begin{tabular}{c|}
$\begin{array}{c}\text { Before } \\
\text { curing }\end{array}$ \\
32.2 \\
\end{tabular}} & \multirow{2}{*}{$\begin{array}{c}\begin{array}{c}\text { After } \\
\text { curing }\end{array} \\
46.9 \\
\end{array}$} & \multirow{2}{*}{$\begin{array}{c}\begin{array}{c}\text { Before } \\
\text { curing }\end{array} \\
45.8 \\
\end{array}$} & \multirow{2}{*}{$\begin{array}{c}\begin{array}{c}\text { After } \\
\text { curing }\end{array} \\
78.4 \\
\end{array}$} & \multirow{2}{*}{$\begin{array}{c}\begin{array}{c}\text { Before } \\
\text { curing }\end{array} \\
83.2 \\
\end{array}$} & \multirow{2}{*}{$\begin{array}{c}\begin{array}{c}\text { After } \\
\text { curing }\end{array} \\
58.5 \\
\end{array}$} \\
\hline$D E_{\max }(\%)$ & Experimental & & & & & & \\
\hline$D E_{\max }(\%)$ & Fitted & 32.2 & 46.3 & 45.9 & 78.5 & 84.0 & 59.1 \\
\hline$\Delta n\left(\times 10^{-3}\right)$ & Fitted & 0.9 & 1.2 & 1.3 & 1.9 & 2.4 & 3.4 \\
\hline$d$ & Fitted & 131.5 & 117.8 & 141.2 & 132.8 & 128.8 & 118.1 \\
\hline$\Delta n \times d(\mu \mathrm{m})$ & Calculated & 0.118 & 0.141 & 0.184 & 0.252 & 0.309 & 0.402 \\
\hline$n$ & Fitted & 1.499 & 1.505 & 1.507 & 1.507 & 1.500 & 1.501 \\
\hline
\end{tabular}

\section{CONCLUSIONS}

The most remarkable result is the demonstration of more than a $30 \% \mathrm{DE}$ increment of non-overmodulated holographic gratings after a curing treatment to stabilize the hologram over time. The gratings were stored in a low-toxicity photopolymer and the curing stage was performed with an incoherent and low-cost LED lamp. The experimental results were also supported by theoretical adjustments based on the Kogelnik's Coupled Wave Theory.

\section{ACKNOWLEDGMENTS}

Ministerio de Ciencia, Innovación y Universidades, Spain, under projects FIS2017-82919-R (MINECO/AEI/FEDER, UE) and FIS2015-66570-P (MINECO/FEDER); Generalitat Valenciana, Spain, under project CDEIGENT/2018/024 and GRE17-06.

\section{REFERENCES}

[1] Ortuño, M., Fernández, E., Gallego, S., Beléndez, A. and Pascual, I., "New photopolymer holographic recording material with sustainable design," Opt. Express 15(19), 12425-12435 (2007).

[2] Ortuño, M., Gallego, S., Márquez, A., Neipp, C., Pascual, I. and Beléndez, A., "Biophotopol: A sustainable photopolymer for holographic data storage applications," Materials 5(12), 772-783 (2012).

[3] Navarro-Fuster, V., Ortuño, M., Gallego, S., Márquez, A., Beléndez, A. and Pascual, I., "Biophotopol's energetic sensitivity improved in $300 \mu \mathrm{m}$ layers by tuning the recording wavelength," Opt. Mater. 52, 111-115 (2016).

[4] Ramírez, M. G., Sirvent, D., Morales-Vidal, M., Ortuño, M., Martínez-Guardiola, F. J. and Pascual, I., "LEDcured reflection gratings stored in an acrylate-based photopolymer," Polymers 11(632), 632 (2019).

[5] Lloret, T., Navarro-Fuster, V., Ramírez, M. G., Ortuño, M., Neipp, C., Beléndez, A. and Pascual, I., "Holographic lenses in an environment-friendly photopolymer," Polymers 10(302), (2018).

[6] Fernández, E., Fuentes, R., Ortuño, M., Beléndez, A. and Pascual, I., "Holographic grating stability: influence of 4,4'-azobis (4-cyanopentanoic acid) on various spatial frequencies," Appl. Opt. 52(25), 6322-6331 (2013).

[7] Gallego, S., Neipp, C., Ortuñto, M., Beléndez, A. and Pascual, I., "Stabilization of volume gratings recorded in polyvinyl alcohol-acrylamide photopolymers with diffraction efficiencies higher than 90\%," J. Mod. Opt. 51(4), 491-503 (2004).

[8] Gallego, S., Ortuño, M., Neipp, C., García, C., Beléndez, A. and Pascual, I., "Overmodulation effects in volume holograms recorded on photopolymers," Opt. Commun. 215(4-6), 263-269 (2003).

[9] Kogelnik, H., "Coupled wave theory for thick hologram gratings," Bell Syst. Tech. J. 48(9), 2909-2947 (1969). 